\title{
Validation of the EUTOS Long-Term Survival Score in Chinese Chronic Myeloid Leukemia Patients Treated with Imatinib: A Multicenter Real-World Study
}

This article was published in the following Dove Press journal: Cancer Management and Research

\section{Xiawan Yang $\mathbb{D}^{1, *}$ \\ Yanliang Bai ${ }^{1, *}$ \\ Mingyue Shi (D) \\ Wanjun Zhang' \\ Junwei Niu' \\ Chengye $\mathrm{Wu}^{2}$ \\ Lei Zhang ${ }^{2}$ \\ Zhiwei $\mathrm{Xu}^{3}$ \\ Xiang Liu $^{4}$ \\ Yuqing Chen' \\ Kai Sun'}

'Department of Hematology, Zhengzhou University People's Hospital and Henan Provincial People's Hospital, Henan, People's Republic of China; ${ }^{2}$ Institute of Hematology, Zhengzhou University People's Hospital and Henan Provincial People's Hospital, Henan, People's Republic of China; ${ }^{3}$ Department of Clinical Research Service Center, Zhengzhou University People's Hospital and Henan Provincial People's Hospital, Henan, People's Republic of China; ${ }^{4}$ Department of Geriatric Medicine, Zhengzhou University People's Hospital and Henan Provincial People's Hospital, Henan, People's Republic of China

*These authors contributed equally to this work

Correspondence: Kai Sun; Yuqing Chen Department of Hematology, Zhengzhou University People's Hospital and Henan Provincial People's Hospital, No. 7 Weiwu Road, Jinshui District Zhengzhou, Henan 450003, People's Republic of China

Tel +86-I8237II0038; +86- I869732I 863

Email sunkai@cellscience.org;

henanblood@sina.com
Purpose: To validate the clinical efficacy of the recently developed EUTOS long-term survival (ELTS) score in a real-world setting.

Patients and Methods: A total of 479 chronic myeloid leukemia (CML) patients treated with frontline imatinib between January 2010 and December 2017 were enrolled in this retrospective study. The ELTS score was evaluated on the end-points including complete cytogenetic response (CCyR), progression-free survival (PFS), overall survival (OS) and CML-related death, and the efficiency of the ELTS score was further compared with the historical Sokal, Hasford, EUTOS scores.

Results: With a median follow-up of 69 months (range, 9-112 months), 462 evaluable patients were stratified into the ELTS low-risk $(n=230)$, ELTS intermediate-risk $(n=168)$ and ELTS high-risk $(n=64)$ groups. For the regular assessment indicators like CCyR, PFS and OS, the ELTS scoring system could effectively identify the corresponding risk groups, similarly with the results provided by previous scoring systems. With respect to the CML-related death, the ELTS score could accurately identify a highrisk group with a significantly higher risk of dying of CML, and the 5-year cumulative incidence occurred in the ELTS high-, intermediate-, and low-risk groups was 11\% (95\% CI: 3-19\%), 5\% (95\% CI: 1-9\%) and 2\% (95\% CI: 0-4\%), respectively. Most notably, the ELTS score outperformed the Sokal, Hasford and EUTOS scores without statistical difference among different risk groups.

Conclusion: The ELTS score could effectively predict the prognosis of imatinib-treated CML patients in real-life settings.

Keywords: chronic myeloid leukemia, tyrosine kinase inhibitors, Sokal, Hasford, EUTOS, EUTOS long-term survival score

\section{Introduction}

Chronic myeloid leukemia (CML), a clonal myeloproliferative neoplasm, is characterized by the oncogenic BCR-ABL fusion gene that encodes constitutive tyrosine kinase activity. ${ }^{1,2}$ Compared with the interferon and intensive chemotherapy, the imatinib, a first-generation tyrosine kinase inhibitor as first-line therapy, greatly improved the survival rate from $57 \%$ and $42 \%$ to $83-89 \%$. $^{3-6}$ What comes with this improvement is that the lifetime expectancy of CML patients gradually reaches a comparable stage to that of the general population. ${ }^{7,8}$ The major causes of death are no longer CML-related. Thus, taking overall survival (OS), irrespective of the causes of death, as the prognostic indicator was challenged. Instead, CML-related death 
could be a better assessment of treatment efficacy, which make establishing a reliable prognostic model for predicting the risk of disease-related death imperative.

Historically, the Sokal and Hasford scores were performed in the intensive chemotherapy and interferon era, using mathematical equations including age, spleen size, platelets and blasts for the Sokal score; basophils and eosinophil cell count in blood as supplementary biological parameters for the Hasford score. ${ }^{9,10}$ Subsequently, the European Treatment and Outcome Study (EUTOS) score, which obviously simplified the calculation formula by using only basophils and spleen size, was introduced in the imatinib era. ${ }^{11}$ The above scores were widely implemented to evaluate treatment response and long-term outcome of CML patients receiving defined treatment. ${ }^{6,12-15}$ However, there lacked the risk prediction in disease-specific mortality. In 2016, the EUTOS long-term survival (ELTS) score was proposed in a clinical study enrolling a cohort of 2290 imatinib-treated patients with chronic-phase CML (CML-CP) diagnosed between 2002 and 2006 and showed a better ability to determine the probability of dying from CML. ${ }^{16}$

Recently, the new ELTS score has been validated in Europe to further clarify the clinical significance. ${ }^{16-18}$ However, both clinical trials and real-word studies with Asian populations remain deficient, especially Chinese populations. Therefore, we performed a retrospective study to validate the predictive ability of the ELTS scoring system with Chinese CML-CP patients. In addition, the previous scoring methods, including the Sokal, Hasford and EUTOS scores, were analyzed and compared.

\section{Patients and Methods}

\section{Patients}

From January 2010 to December 2017, 479 adult patients newly diagnosed with Philadelphia chromosome-positive and/or BCR-ABL-positive CML-CP treated with imatinib as initial therapy at Zhengzhou University People's Hospital and Hospital League were enrolled in this retrospective study. All baseline characteristics were extracted from the medical records, including age, sex, spleen size, white blood cell count, hemoglobin, platelet count, and the percentage of blasts, basophils and eosinophils in peripheral blood. Then, we evaluated the prognostic efficiency of each four scoring systems for time to first Complete cytogenetic response (CCyR) attainment, progression-free survival (PFS), OS and CML-related death. The study protocol was conducted in accordance with the Declaration of Helsinki, and the protocol was approved by the research ethics committees of each institution. Each participant signed a written informed consent form before enrollment.

\section{Definitions}

The ELTS risk score was determined by age, spleen size, platelets and blasts count. Details on calculation formulas of the four scoring systems are summarized in Table 1. Progression was described as disease development of accelerated phase and blastic phase according to the European Leukemia Net (ELN) criteria. ${ }^{19}$ CML-related death was measured from the date that imatinib treatment started to the date of disease-specific death, defined as death after recorded CML progression. The final follow-up for all patients occurred on May 1, 2019.

\section{Statistical Analysis}

The cumulative incidence of all four endpoints was estimated by the Kaplan-Meier method, and the differences among different risk stratifications were compared by the Log-rank test. The data were analyzed using SPSS software (SPSS 24.0 for Windows, IL, U.S.A.). A $p$ value $<$ 0.05 was considered statistically significant.

Table I Calculation Formulas of Each Scoring System: The Sokal, Hasford, EUTOS and ELTS Scores

\begin{tabular}{|c|c|c|}
\hline $\begin{array}{l}\text { Scoring } \\
\text { System }\end{array}$ & Calculation & Risk Definition \\
\hline $\begin{array}{l}\text { Sokal } \\
\text { score }\end{array}$ & $\begin{array}{l}\operatorname{Exp} 0.0116 \times(\text { age }-43.4)+0.0345 \times \\
(\text { spleen }-7.51)+0.1880 \times[(\text { platelet } \\
\left.\text { count } / 700)^{2}-0.563\right]+0.0887 \times(\text { blasts }- \\
2.10)\end{array}$ & $\begin{array}{l}\text { Low risk: }<0.8 \\
\text { Intermediate risk: } \\
0.8-1.2 \\
\text { High risk: }>1.2\end{array}$ \\
\hline $\begin{array}{l}\text { Hasford } \\
\text { score }\end{array}$ & $\begin{array}{l}(0.6666 \times \text { age }[0 \text { when age }<50 \text { years; } I \text {, } \\
\text { otherwise }]+0.0420 \times \text { spleen }+0.0584 \times \\
\text { blasts }+0.0413 \times \text { eosinophils }+0.2039 \times \\
\text { basophils }[0 \text { when basophils }<3 \% ; 1 \text {, } \\
\text { otherwise }]+1.0956 \times \text { platelet count }[0 \\
\text { when platelets }<1500 \times 10^{9} / L ; \text { I, } \\
\text { otherwise }]) \times 1000\end{array}$ & $\begin{array}{l}\text { Low risk: } \leq 780 \\
\text { Intermediate risk: } \\
78 \mid-1480 \\
\text { High risk: }>1480\end{array}$ \\
\hline $\begin{array}{l}\text { EUTOS } \\
\text { score }\end{array}$ & $($ Basophils $\times 7)+($ spleen $\times 4)$ & $\begin{array}{l}\text { Low risk: } \leq 87 \\
\text { High risk: }>87\end{array}$ \\
\hline $\begin{array}{l}\text { ELTS } \\
\text { score }\end{array}$ & $\begin{array}{l}0.0025 \times(\text { age } / 10)^{3}+0.0615 \times \text { spleen }+ \\
0.1052 \times \text { blasts }+0.4104 \times(\text { platelet } \\
\text { count } / 1000)^{-0.5}\end{array}$ & $\begin{array}{l}\text { Low risk: } \leq 1.5680 \\
\text { Intermediate risk: } \\
\text { I.5680-2.2185 } \\
\text { High risk: }>2.2185\end{array}$ \\
\hline
\end{tabular}

Note: Exp, exponential function; Age is in years; Spleen is in $\mathrm{cm}$ below the costal margin; Platelet count is in $\times 10^{9} / \mathrm{L}$; Blasts, eosinophils and basophils are in percent of peripheral blood.

Abbreviations: EUTOS, European Treatment and Outcome Study; ELTS, EUTOS long-term survival. 


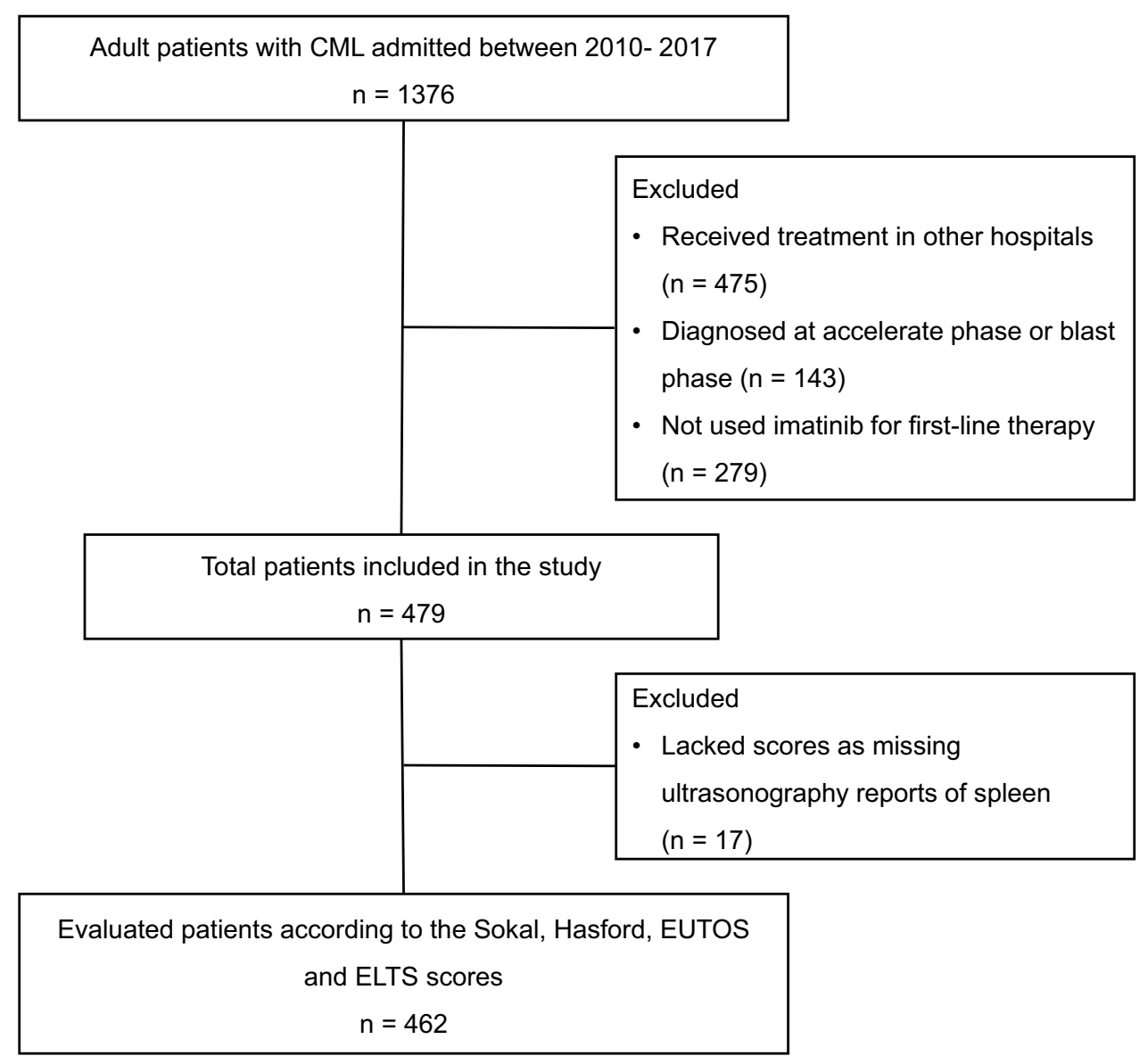

Figure I Study flow-chart.

\section{Results}

\section{Baseline Characteristics}

We identified 479 patients newly diagnosed with CML-CP and using imatinib as the first-line treatment. The study flow-chart is shown in Figure 1. The median follow-up duration of the surviving patients was 69 months (range, 9-112 months). The patients' median age at diagnosis was 49 years (range, 18-86 years). Splenomegaly was observed in $62.8 \%$ of the patients, and the median spleen size below the costal margin was $8 \mathrm{~cm}$ (range, 0 to $21 \mathrm{~cm}$ ), which showed there was a higher proportion of patients with splenomegaly and the larger median spleen size in our CML cohort. The detail baseline characteristics of patients are shown in Table 2.

\section{Risk Stratification}

A total of 462 evaluable patients were divided into the discordant risk categorizations for the lack of ultrasonography reports in 17 patients. Data on risk stratification demonstrated that more intermediate- and high-risk patients classified by the Sokal and Hasford scores were allocated into the ELTS low-risk group. The proportion of the ELTS high-risk group was similar to that in the Hasford and EUTOS high-risk groups, but obviously

Table 2 Patient Demographics and Baseline Characteristics

\begin{tabular}{|l|l|}
\hline Clinical Characteristics & Median (Range) or n (\%) \\
\hline Age, year & $49(18-86)$ \\
\hline Sex & \\
$\quad$ Male (\%) & $270(56.4 \%)$ \\
Female (\%) & $209(43.6 \%)$ \\
\hline White blood cell count, $\times 10^{9} / \mathrm{L}$ & $90.27(3.5 \mathrm{I}-626.42)$ \\
Hemoglobin, g/L & $103(47-15 \mathrm{I})$ \\
Platelet count, $\times 10^{9} / \mathrm{L}$ & $473(32-2198)$ \\
Eosinophils, \% in peripheral blood & $3(0-21)$ \\
Basophils, \% in peripheral blood & $4(0-17)$ \\
Blasts, \% in peripheral blood & $0(0-9)$ \\
Blasts, \% in bone marrow & $3(0-12)$ \\
Spleen enlargement & $301(62.8 \%)$ \\
Spleen size below the costal margin, $\mathrm{cm}$ & $8(0-21)$ \\
\hline
\end{tabular}


Table 3 The Distribution of CML Patients Risk Stratified According to Each of the Four Scoring Systems

\begin{tabular}{|l|l|l|l|l|}
\hline Risk Groups & $\begin{array}{l}\text { Sokal } \\
\text { Score }\end{array}$ & $\begin{array}{l}\text { Hasford } \\
\text { Score }\end{array}$ & $\begin{array}{l}\text { EUTOS } \\
\text { Score }\end{array}$ & $\begin{array}{l}\text { ELTS } \\
\text { Score }\end{array}$ \\
\hline $\begin{array}{l}\text { Low, n (\%) } \\
\text { Intermediate, n } \\
(\%)\end{array}$ & $122(26.4 \%)$ & $135(29.2 \%)$ & $411(89.0 \%)$ & $230(49.8 \%)$ \\
High, n (\%) & $141(30.5 \%)$ & $61(13.2 \%)$ & $51(11.0 \%)$ & $64(13.8 \%)$ \\
\hline
\end{tabular}

Abbreviations: EUTOS, European Treatment and Outcome Study; ELTS, EUTOS long-term survival.

lower than the Sokal high-risk group. The details about the distribution of risk subgroups via each of the four score systems are described in Table 3. According to the ELTS score, 230 patients $(49.8 \%)$ were determined to be at low risk, while 168 (36.4\%) and 64 patients (13.8\%) were stratified as intermediate and high risk, respectively. Using the Sokal and Hasford scores, $122(26.4 \%)$ and $135(29.2 \%)$ patients, $199(43.1 \%)$ and 266 (57.6\%) patients and $141(30.5 \%)$ and $61(13.2 \%)$ patients were categorized as low risk, intermediate risk and high risk, respectively. The distribution according to the EUTOS score was 411 patients $(89.0 \%)$ in the low-risk group and $51(11.0 \%)$ in the high-risk group.

\section{Verification Results for CCyR}

CCyR was considered as an early surrogate factor for assessing CML response to imatinib treatment. We choose CCyR within 18 months as endpoint events to compare the predictive abilities of each scoring systems. The ELTS score could clearly discriminate the low-risk group, comparing with the high-risk ( $88 \%$ vs $66 \%, p<0.001)$ and intermediate-risk groups $(88 \%$ vs $72 \%, p<0.001)$ (Figure 2A), which was the roughly same as that of the Hasford score described. By comparison, the Sokal score was only able to differentiate between low-risk and intermediate-risk groups. The EUTOS scoring model had no predictive capabilities in distinguishing the cumulative incidence of first CCyR attainment (Figure 2B-D).
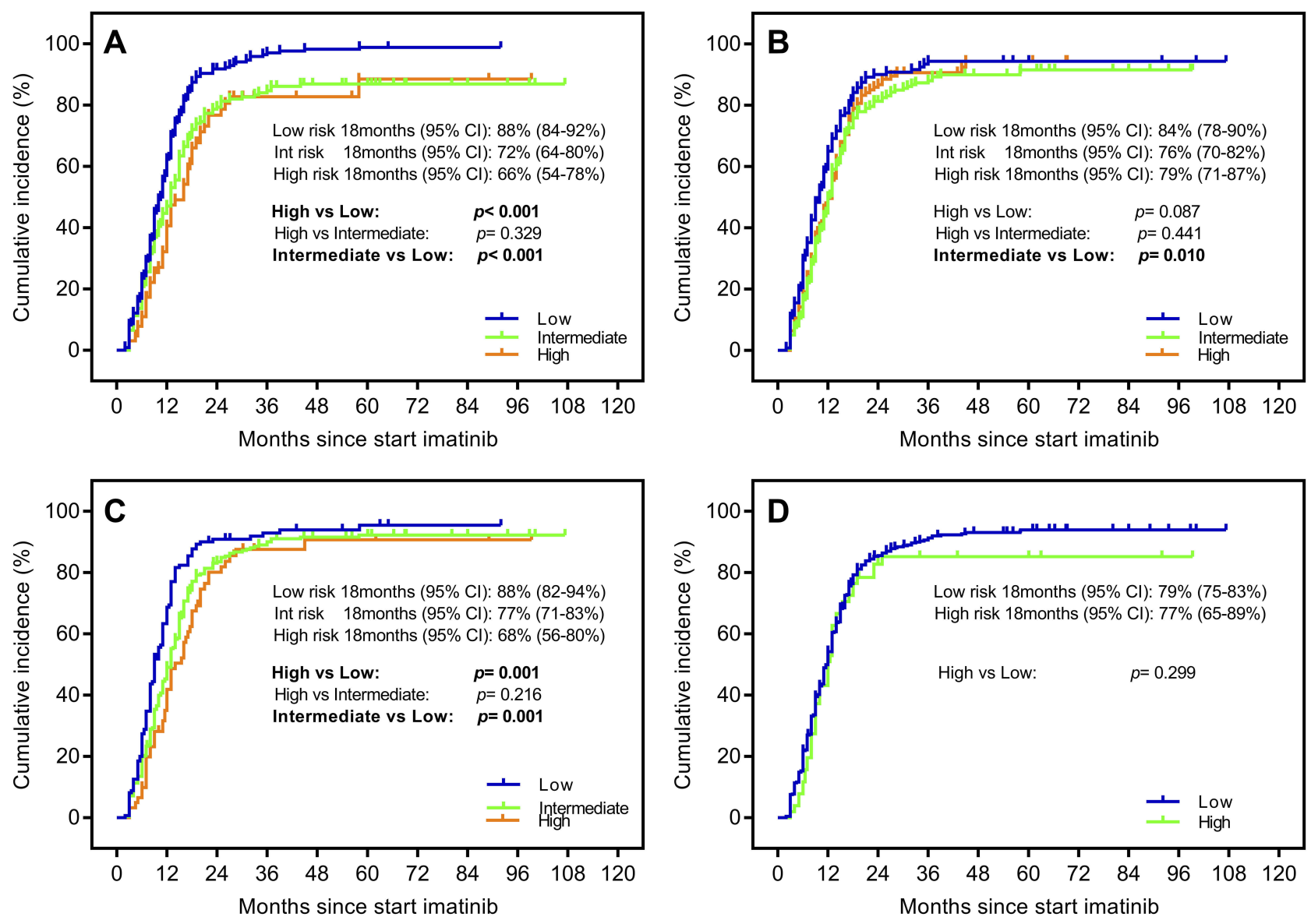

Figure 2 Probability of achieving complete cytogenetic response (CCyR) stratified by (A) the EUTOS long-term survival (ELTS) score and (B) the Sokal score, (C) the Hasford score and (D) the European Treatment and Outcome Study (EUTOS) score. 


\section{Verification Results for Survival Time}

Due to the improvement of CML survival time in the imatinib era, we prolonged the follow-up time and took the occurrence of progression or death within 5 years as end points for evaluating PFS and OS. In terms of PFS, estimated 5-year probabilities of 93\% (95\% CI: 89-97\%), 84\% (95\% CI: $78-90 \%)$ and $83 \%$ (95\% CI: 73-93\%) were shown in the low-, intermediate- and high-risk groups according to the ELTS score. The data illustrated that the ELTS prediction model identified a low-risk group with a lower risk rate comparing with the intermediate- and highrisk groups $(p=0.013$ and $p=0.022$, respectively) (Figure 3A). Verification results of previous scoring systems showed that only EUTOS was capable of identifying risk groups with significantly different survival probabilities. The Sokal and Hasford scores both did not identify different risk groups (Figure 3B-D). With respect to OS, the 5-year survival rates were $98 \%$ (95\% CI: $96-100 \%)$, 89\% (95\% CI: $83-95 \%)$ and 79\% (95\% CI: $78-91 \%)$ in the low-, intermediate- and high-risk groups, respectively
( $p=0.084, p<0.001$ and $p=0.009$, respectively) (Figure 4A). The verification data from four scoring models demonstrated that the ELTS, Sokal and EUTOS scores, except for the Hasford score, could discriminate a high-risk group, which existed a significantly higher risk of dying from all causes (Figure 4B-D).

\section{Verification Results for CML-Related Death}

The indicator of CML-related death was proposed to explore disease-specific mortality, which was never taken into account via the previous score system. Among the evaluated patients, death due to CML occurred in 17 patients: the ELTS low-, intermediate- and high-risk groups were recorded in 4 (1.74\%), 7 (4.17\%), $6(9.38 \%)$ patients, respectively. The ELTS score divided the CML patients into separate risk subgroups with the high-risk group differing significantly from the other risk groups, and the survival analysis showed the 5year cumulative incidence of CML-related death in the high-, intermediate- and low-risk group was $11 \%$ (95\% CI: 3-19\%), 5\% (95\% CI: $1-9 \%)$ and 2\% (95\% CI: 0-4\%), respectively
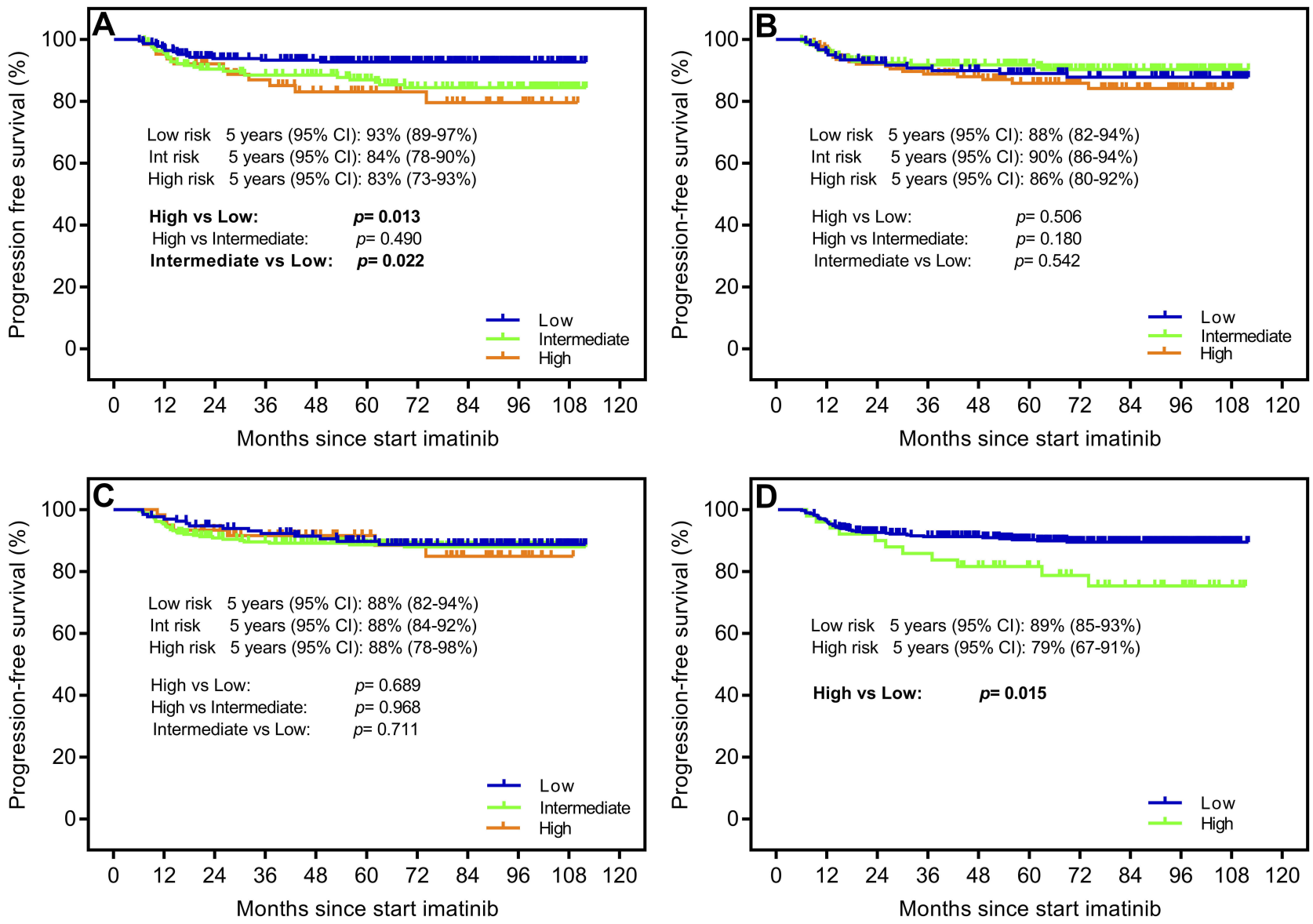

Figure 3 Probability of progression-free survival (PFS) stratified by (A) the EUTOS long-term survival (ELTS) score, (B) the Sokal score, (C) the Hasford score and (D) the European Treatment and Outcome Study (EUTOS) score. 

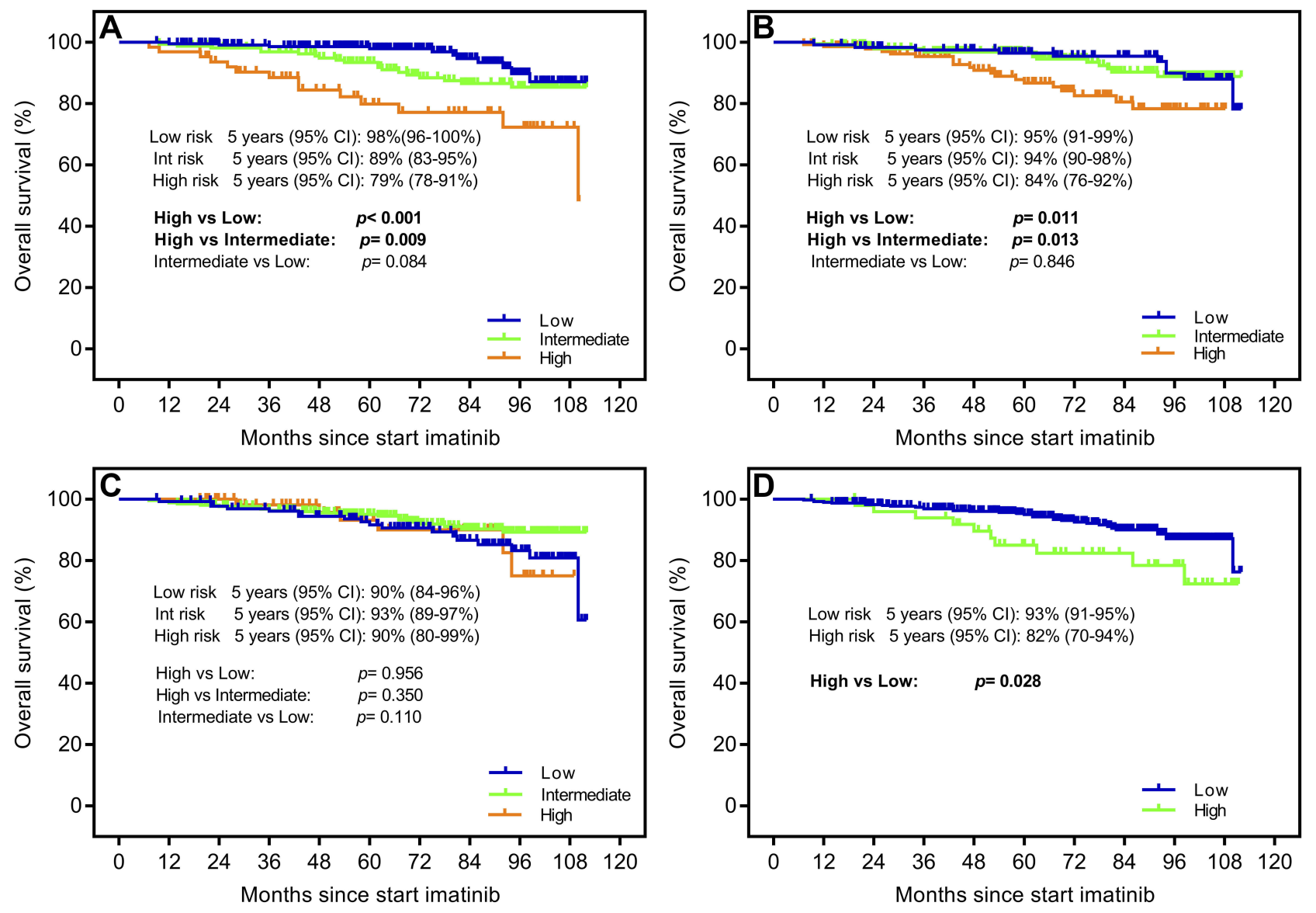

Figure 4 Probability of overall survival (OS) stratified by (A) the EUTOS long-term survival (ELTS) score, (B) the Sokal score, (C) the Hasford score and (D) the European Treatment and Outcome Study (EUTOS) score.

$(p=0.002, p=0.043$ and $p=0.192)$ (Figure 5A). In contrast, the Sokal, Hasford and EUTOS risk scores all failed to distinguish the cumulative incidence of dying from CML (Figure 5B-D).

\section{Discussion}

Prognostic scores are established to predict the prognosis of CML patients presenting in chronic phase. Based on the improved survival time in imatinib era, nearly half of the deaths are not directly related to CML. ${ }^{7,16,20}$ Thus, the ELTS score first considered the disease-related death substituting for OS as evaluation indicators. To further explore the predictive value of the ELTS score in China, we performed a multicenter retrospective study.

The main factors of the ELTS score are similar to that of the Sokal and Hasford scores, incorporating the age, spleen size, platelets and blasts count. ${ }^{9,10,16}$ In our present cohort of 479 adult CML-CP patients treated with imatinib, the ELTS score identified a similar proportion of high-risk patients to the Hasford and EUTOS scores, but obviously lower proportion than the Sokal score, while the ratios of patients allocated to low-risk (49.8\%), intermediate-risk (36.4\%) and high-risk (13.9\%) groups were consistent with previously published reports. ${ }^{11,16,18,21}$

We observed the rate of cytogenetic remissions and survival time, as the regular assessment indicators reported in the original researches. For CCyR, over half of the patients could achieve cytogenetic remission within 18 months. With respect to the survival time including PFS and OS, the ELTS score could effectively identify the corresponding risk group. The whole data compared favorably with the results in prior reports. ${ }^{11,14,21-25}$

The cumulative 5-year CML-related death rate reported in our research was $11 \%$ in the ELTS high-risk group, $5 \%$ in the ELTS intermediate-risk group, and $2 \%$ in the ELTS lowrisk group. The data demonstrated there was higher incidence compared with the results reported in the confirmatory study by Geelen et al. ${ }^{18}$ Although the incidence was not exactly consistent, the ELTS score still distinguished the high-risk group with a significantly higher rate of disease-related death than the other risk groups. Most notably, the ELTS score 

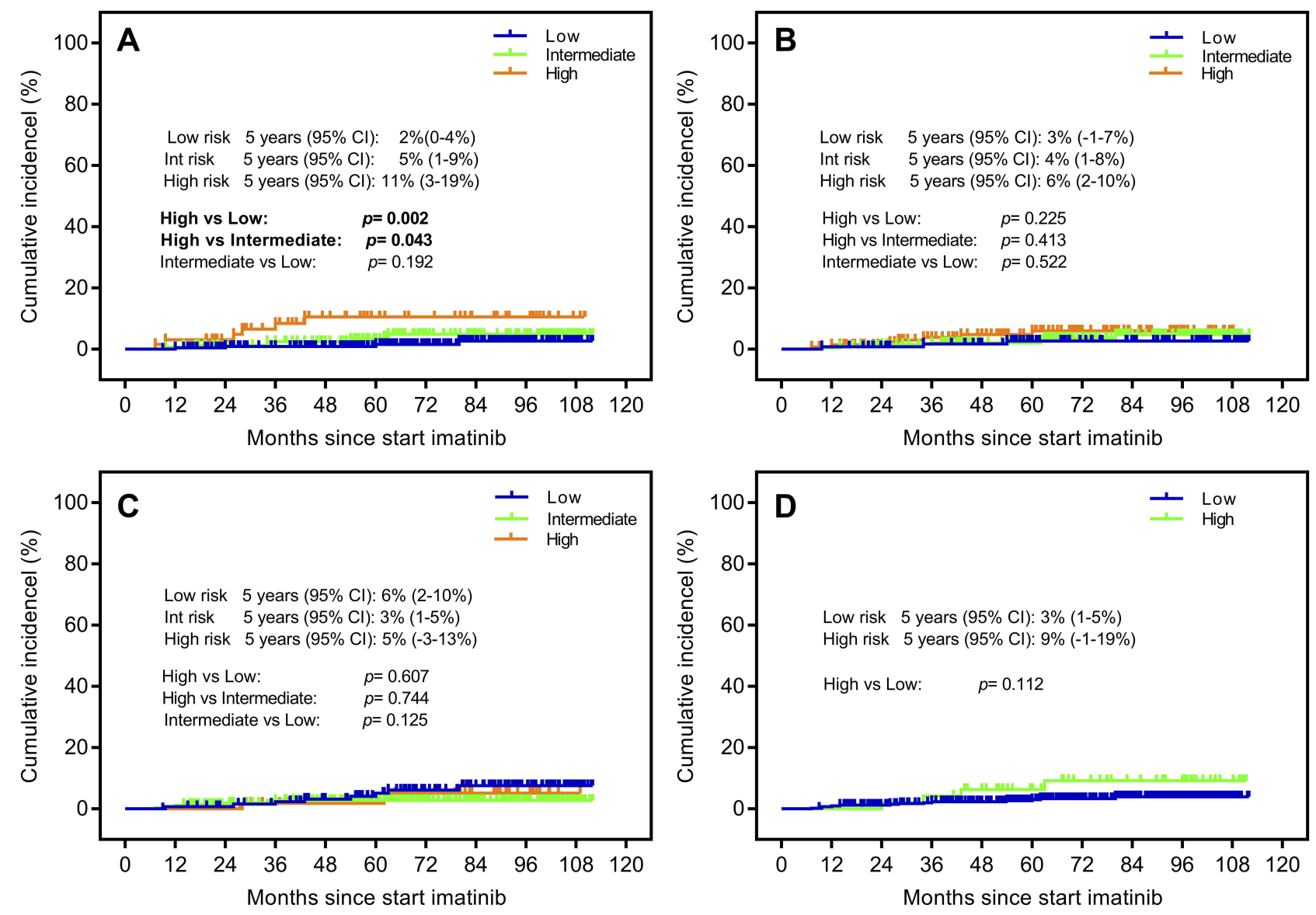

Figure 5 Probability of CML-related death stratified by (A) the EUTOS long-term survival (ELTS) score, (B) the Sokal score, (C) the Hasford score and (D) the European Treatment and Outcome Study (EUTOS) score.

outperformed the previously established Sokal, Hasford and EUTOS scores without statistical difference among different risk groups. Those findings suggest to tailor personalized therapy absorbing this new scoring system into the therapeutic algorithms of the current recommendations. The high incidence of disease-related death for patients distributed to the ELTS high-risk group could favor risk-adjusted treatment with the application of second-generation tyrosine kinase inhibitors as the frontline therapy in these patients. Besides, the low rate of disease-specific mortality in the ELTS lowrisk group might provide additional references to imatinib discontinuation in CML patients with good therapeutic effect. Actually, the usefulness of the ELTS score is not certain. Few studies have validated the effectiveness of the ELTS score in clinical trials or in real-life setting. ${ }^{17,18,21}$ Our study strongly provided positive evidence to support that the ELTS score is a significant risk stratification measure for CML-CP patients treated with imatinib.

However, there are certain limitations in our real-world study. Among the evaluated CML patients, some patients reduced their dosage or discontinued tyrosine kinase inhibitor therapy owing to economic stress, imatinib intolerance or resistance. ${ }^{26}$ Poor adherence to treatment is a considerable reason for not achieving CCyR, disease progression, or even dying from CML. 19,27,28

\section{Conclusion}

Our results implied that the new ELTS score may be an alternative method to evaluate prognosis, especially in the risk of CML-related death. In turn, these improvements in the disease assessment will help clinicians to better forecast treatment challenges and promote the optimization of future treatment paradigms. We, therefore, suggest that the ELTS score should be applied in clinical practice.

\section{Abbreviations}

EUTOS, European Treatment and Outcome Study; ELTS, EUTOS long-term survival; CML, chronic myeloid leukemia; CCyR, complete cytogenetic response; PFS, progression-free 
survival; OS, overall survival; CML-CP, chronic-phase CML; ELN, European Leukemia Net; CI, confidence interval.

\section{Ethics Approval and Consent to Participate}

The study protocol was approved by the research ethics committees of Zhengzhou university people's hospital and hospital league. Each participant signed a written informed consent form before enrolment.

\section{Acknowledgments}

We thank Drs. Honggang Guo and Xiaona Niu for their assistance with performing the clinical research. We thank Drs. Yanru Pei and Juanjuan Song for summarizing and analyzing the data. We thank Drs. Shengjie Wei, Mengyu Xiao, Liurui Dou and Dan Li for collecting vital clinical data. We thank all the hospitals and hematology departments who participated in this real-world survey. We also appreciate the work of data managers in the institutes of hematology and ultrasonography departments.

\section{Funding}

This study was partially supported by grants from the National Natural Science Foundation of China (No. 81971508, No. 81471589, No. 81273259).

\section{Disclosure}

The authors declare no conflicts of interest in this work.

\section{References}

1. Deininger MW, Vieira S, Mendiola R, et al. BCR-ABL tyrosine kinase activity regulates the expression of multiple genes implicated in the pathogenesis of chronic myeloid leukemia. Cancer Res. 2000;60 (7):2049-2055.

2. Maru Y. Molecular biology of chronic myeloid leukemia. Cancer Sci. 2012;103(9):1601-1610. doi:10.1111/j.1349-7006.2012.02346.x

3. Sawyers CL. Chronic myeloid leukemia. N Engl J Med. 1999;340 (17):1330-1340. doi:10.1056/NEJM199904293401706

4. Chronic Myeloid Leukemia Trialists' Collaborative Group. Interferon alfa versus chemotherapy for chronic myeloid leukemia: a meta-analysis of seven randomized trials. J Natl Cancer Inst. 1997;89 (21):1616-1620. doi:10.1093/jnci/89.21.1616

5. Druker BJ, Guilhot F, O'Brien SG, et al. Five-year follow-up of patients receiving imatinib for chronic myeloid leukemia. $N$ Engl $J$ Med. 2006;355(23):2408-2417. doi:10.1056/NEJMoa062867

6. de Lavallade H, Apperley JF, Khorashad JS, et al. Imatinib for newly diagnosed patients with chronic myeloid leukemia: incidence of sustained responses in an intention-to-treat analysis. $J$ Clin Oncol. 2008;26(20):3358-3363. doi:10.1200/JCO.2007.15.8154

7. Bower H, Bjorkholm M, Dickman PW, et al. Life expectancy of patients with chronic myeloid leukemia approaches the life expectancy of the general population. J Clin Oncol. 2016;34(24):2851-2857. doi:10.1200/JCO.2015.66.2866
8. Hoffmann VS, Baccarani M, Hasford J, et al. Treatment and outcome of 2904 CML patients from the EUTOS population-based registry. Leukemia. 2017;31(3):593-601. doi:10.1038/leu.2016.246

9. Sokal JE, Cox EB, Baccarani M, et al. Prognostic discrimination in "good-risk" chronic granulocytic leukemia. Blood. 1984;63(4):789799. doi:10.1182/blood.V63.4.789.789

10. Hasford J, Pfirrmann M, Hehlmann R, et al. A new prognostic score for survival of patients with chronic myeloid leukemia treated with interferon alfa. Writing committee for the collaborative CML prognostic factors project group. J Natl Cancer Inst. 1998;90(11):850858. doi: $10.1093 /$ jnci/90.11.850

11. Hasford J, Baccarani M, Hoffmann V, et al. Predicting complete cytogenetic response and subsequent progression-free survival in 2060 patients with CML on imatinib treatment: the EUTOS score. Blood. 2011;118(3):686-692. doi:10.1182/blood-2010-12-319038

12. Bonifazi F, De Vivo A, Rosti G, et al. Testing Sokal's and the new prognostic score for chronic myeloid leukaemia treated with alphainterferon. Italian Cooperative Study Group on Chronic Myeloid Leukaemia. Br J Haematol. 2000;111(2):587-595. doi:10.1046/ j.1365-2141.2000.02366.x

13. Hochhaus A, O'Brien SG, Guilhot F, et al. Six-year follow-up of patients receiving imatinib for the first-line treatment of chronic myeloid leukemia. Leukemia. 2009;23(6):1054-1061. doi:10.1038/ leu. 2009.38

14. Xia L, Qian W, Yang M, et al. Comparison of the utility and applicability of the Sokal, Hasford, and EUTOS scores in a population of Chinese patients with chronic-phase chronic myeloid leukemia undergoing imatinib therapy. Onco Targets Ther. 2015;8:24852492. doi:10.2147/OTT.S 85313

15. Francis J, Dubashi B, Sundaram R, et al. Influence of Sokal, Hasford, EUTOS scores and pharmacogenetic factors on the complete cytogenetic response at 1 year in chronic myeloid leukemia patients treated with imatinib. Med Oncol. 2015;32(8):213. doi:10.1007/s12032-015-0665-0

16. Pfirrmann M, Baccarani M, Saussele S, et al. Prognosis of long-term survival considering disease-specific death in patients with chronic myeloid leukemia. Leukemia. 2016;30(1):48-56. doi:10.1038/ leu.2015.261

17. Molica M, Canichella M, Alunni Fegatelli D, et al. The Eutos longterm survival score accurately predicts the risk of death in chronic myeloid leukaemia patients treated outside of clinical trials. Am J Hematol. 2017;92(12):E661-E664. doi:10.1002/ajh.v92.12

18. Geelen IGP, Sandin F, Thielen N, et al. Validation of the EUTOS long-term survival score in a recent independent cohort of "real world" CML patients. Leukemia. 2018;32(10):2299-2303. doi:10.1038/s41375-018-0136-7

19. Baccarani M, Deininger MW, Rosti G, et al. European LeukemiaNet recommendations for the management of chronic myeloid leukemia: 2013. Blood. 2013;122(6):872-884. doi:10.1182/blood-2013-05-501569

20. Hehlmann R, Muller MC, Lauseker M, et al. Deep molecular response is reached by the majority of patients treated with imatinib, predicts survival, and is achieved more quickly by optimized highdose imatinib: results from the randomized CML-study IV. $J$ Clin Oncol. 2014;32(5):415-423. doi:10.1200/JCO.2013.49.9020

21. Millot F, Guilhot J, Suttorp M, et al. Prognostic discrimination based on the EUTOS long-term survival score within the International Registry for Chronic Myeloid Leukemia in children and adolescents. Haematologica. 2017;102(10):1704-1708. doi:10.3324/ haematol.2017.170035

22. Geelen IGP, Thielen N, Janssen J, et al. Treatment outcome in a population-based, 'real-world' cohort of patients with chronic myeloid leukemia. Haematologica. 2017;102(11):1842-1849. doi:10.33 24/haematol.2017.174953

23. Langabeer SE, Gale RE, Harvey RC, et al. Transcription-mediated amplification and hybridisation protection assay to determine BCRABL transcript levels in patients with chronic myeloid leukaemia. Leukemia. 2002;16(3):393-399. doi:10.1038/sj.leu.2402392 
24. Tao Z, Liu B, Zhao Y, et al. EUTOS score predicts survival and cytogenetic response in patients with chronic phase chronic myeloid leukemia treated with first-line imatinib. Leuk Res. 2014;38(9):10301035. doi:10.1016/j.leukres.2014.06.006

25. Yamamoto E, Fujisawa S, Hagihara M, et al. European Treatment and Outcome Study score does not predict imatinib treatment response and outcome in chronic myeloid leukemia patients. Cancer Sci. 2014;105(1):105-109. doi:10.1111/cas.12321

26. Noens L, van Lierde MA, De Bock R, et al. Prevalence, determinants, and outcomes of nonadherence to imatinib therapy in patients with chronic myeloid leukemia: the ADAGIO study. Blood. 2009;113 (22):5401-5411. doi:10.1182/blood-2008-12-196543
27. Jabbour E, Saglio G, Radich J, et al. Adherence to BCR-ABL inhibitors: issues for CML therapy. Clin Lymphoma Myeloma Leuk. 2012;12(4):223-229. doi:10.1016/j.clml.2012.04.002

28. Marin D, Bazeos A, Mahon FX, et al. Adherence is the critical factor for achieving molecular responses in patients with chronic myeloid leukemia who achieve complete cytogenetic responses on imatinib. J Clin Oncol. 2010;28(14):2381-2388. doi:10.1200/ JCO.2009.26.3087

\section{Publish your work in this journal}

Cancer Management and Research is an international, peer-reviewed open access journal focusing on cancer research and the optimal use of preventative and integrated treatment interventions to achieve improved outcomes, enhanced survival and quality of life for the cancer patient.
The manuscript management system is completely online and includes a very quick and fair peer-review system, which is all easy to use. Visit http://www.dovepress.com/testimonials.php to read real quotes from published authors. 